\title{
Hitching Healthcare to the Chain: An Introduction to Blockchain Technology in the Healthcare Sector
}

\author{
Mark A. Engelhardt
}

\author{
"Blockchain is a way for people to solve problems by") \\ sharing things.
}

\author{
Diego Espinosa \\ Founder and CEO, Healthcoin
}

\begin{abstract}
Health services must balance patient care with information privacy, access, and completeness. The massive scale of the healthcare industry also amplifies the importance of cost control. The promise of blockchain technology in health services, combined with application layers built atop it, is to be a mechanism that provides utmost privacy while ensuring that appropriate users can easily add to and access a permanent record of information. Blockchains, also called distributed ledgers, enable a combination of cost reduction and increased accessibility to information by connecting stakeholders directly without requirements for third-party brokers, potentially giving better results at lower costs. New ventures are looking to apply blockchain technology to solve real-world problems, including efforts to track public health, centralize research data, monitor and fulfill prescriptions, lower administrative overheads, and organize patient data from an increasing number of inputs. Here, concrete examples of the application of blockchain technology in the health sector are described, touching on near-term promise and challenges.
\end{abstract}

\section{Introduction}

Health is the foundation of an engaged and happy life, and modern humans have been the fortunate beneficiaries of great advances in medical technology (Collins, 2015). With each new technology, more clues become available to decipher the problems that plague our well-being. The advent of individualized information from cheaper genome sequencing, the Internet of Things, and widespread collection of health data may enable researchers to solve formerly inaccessible health problems. However, when this massive quantity of data is spread out with limited access, is in forms not conducive to sharing, cannot be easily packaged for computational methods, or does not exist as a complete record, it is impossible to perform the complex data analysis required to arrive at solutions.

To address these fundamental challenges in health data management, innovators are focusing on four main areas:
1. Putting the patient at the centre. For most patients, sustaining health involves many interactions with a variety of healthcare providers and data collection tools, all of which generate information critical to making informed and appropriate healthcare decisions. There is increasing agreement that information should be available to patients such that they can be active agents in their own care, and patient participation and involvement has become a cornerstone of modern medical practice (Kitson et al., 2013; Stewart, 2001). Caretakers also require access to medical information, however, patients increasingly want to be in control of what information caretakers receive, and under what circumstances.

2. Privacy and access. Equally important to consider is the intimate and highly personal nature of health information. Health information must be private and accessible only by appropriate parties, for appropriate reasons, at appropriate times. Some jurisdictions have legislation in place to protect personal 


\title{
An Introduction to Blockchain Technology in the Healthcare Sector
}

\author{
Mark A. Engelhardt
}

information, (e.g., Canada: Minister of Justice, 2015; United States: Department of Health and Human Services, 2013), which new technologies must take into consideration. Despite the complexity of the problem (de Lusignan et al., 2014; Mold et al., 2012, 2015; Tieu et al., 2016; Woodman et al., 2015), there are efforts underway to enable each adult to have full access to their own medical records (Hankewitz, 2016; Kelsey \& Cavendish, 2014; Suberg, 2017).

3. Completeness of information. Currently, medical information is frequently held by individual providers or private data collectors without full patient access (Das, 2017). This limits the ability of patients to explore options, contribute and correct errors in their own data, and share their information with new practitioners to fully define a medical history. Patientcentred information sharing should enable the patient increased control and better outcomes by ensuring that complete health information is available to the right people at the right time. Lack of information interoperability is detrimental to using new databased diagnostic technologies.

4. Cost. There is a crisis in the cost of healthcare, and expense looms large behind every discussion of changes to its delivery. Health expenditure per capita has increased $60 \%$ over the past 10 years (The World Bank, 2015). In countries such as Australia, the United Kingdom, and Canada, health expenditures represent about $10 \%$ of GDP; in the United States, this number is closer to $17 \%$ (The World Bank, 2015). Paradoxically, outcomes in the United States are worse than elsewhere (Avendano \& Kawachi, 2014), a clear indicator that there is waste in the system. A recent study showed that older people with diagnosed chronic diseases face catastrophic health expenditure even in some of the wealthiest countries in Europe (Arsenijevic et al., 2016). It is of note that the population in developed countries is, on average, aging, and therefore this situation can reasonably be expected to worsen in the future.

Technology can be part of the solution. A study by McKinsey \& Company estimated that more than $\$ 300$ billion could be recovered per year by using health data creatively and effectively, with two-thirds of that in the form of reductions to national health care expenditure - about 8 percent of estimated healthcare spending at 2010 levels (McKinsey \& Company, 2011). In particular, blockchain technology has the potential to hold and control access to massive amounts of anonymized health data, enabling new research and new insights, while at the same time protecting the privacy of patients. Importantly, blockchain technology serves as a protocol to connect important stakeholders to data without requiring an expensive layer of data mediators and escrow services to broker trust, removing middle management and its associated cost from the data-sharing equation. Better data sharing between stakeholders should also reduce waste, for example, that due to duplicate testing that occurs when healthcare providers are not aware of each other's actions.

Don Tapscott, a leader in the industry, has said "though there are many culprits, the root of the problem is our industrial-age thinking about delivering healthcare, where data is hoarded, patients are assumed to be ignorant, and where healthcare is only available when you're in the system. This leads to costly and ineffective care. Blockchain promises to change that. We can fix healthcare by basing it on a set of new principles - collaboration, openness, and integrity, and where the patient co-creates their own data with full transparency into it." (Schumacher, 2017). Blockchain technology is being applied increasingly in the finance sector, but as Mo Tayeb of Medicalchain points out, "your body is more important than your bank account" (personal communication, August 25, 2017). It is now time to take what has been learned and apply it to something even more important: health.

\section{Blockchains are Decentralized, Immutable, Private, and Agents of Trust}

At its core, blockchain technology consists of a few straightforward ideas with interesting properties that align significantly with important healthcare challenges.

Blockchains are distributed ledgers - sequential lists of transactions with identical copies shared and maintained by multiple parties. There is no single source that claims authority over the true data, which is instead declared by consensus amongst the multiple parties holding the data (Figure 1). Because of this, blockchains are referred to as decentralized. This arrangement protects the data from tampering not just by individual keepers of the blockchain, but also external attempts at damage. In one example, the decentralization of blockchain solutions would offer intrinsic protection against assaults such as the recent WannaCry ransomware attacks because the blockchain would only be affected if simultaneously attacked at many sites (Mattei, 2017). 


\section{An Introduction to Blockchain Technology in the Healthcare Sector}

Mark A. Engelhardt

(A)
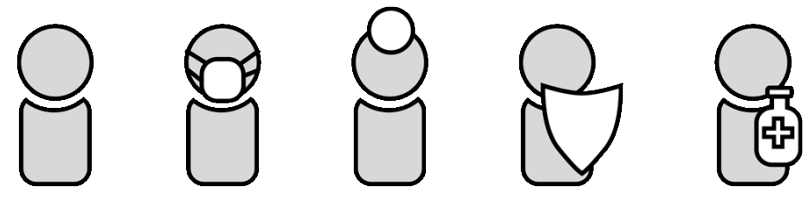

(B)

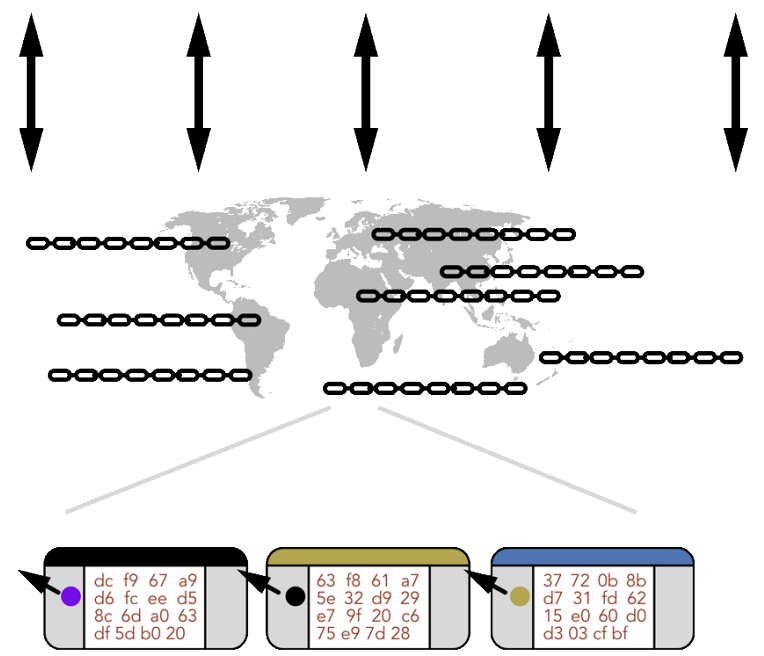

(C)

(D)

Figure 1. Stakeholders (A) have selective and controlled access to data elements stored in a set of identical verified blockchains held at multiple locations (B), wherein each block contains auditable information about creation and sequencing $(\mathrm{C})$ and encrypted private information (D). Information about sequencing could be in the form of a hash that acts as a signature to uniquely describe one or more previous blocks in the chain. Although all arrows between (A) and (B) are shown as double-headed, read and write access to the blockchain would be stakeholder-dependent as defined in smart contracts.

Each record in the chain includes precise information about when it was created and the cryptographic signature of the preceding record in the chain, along with additional arbitrary information. The signature - or hash consists of a cryptographically generated sequence of letters and numbers of a defined length that uniquely identifies any digital entity. Changing any record would change its signature, and would therefore create an easily detectable break in the chain. Records can only be added, never removed, and only by consensus of the maintainers of the distributed copies. Blockchains are thus immutable.

Information in each block can be encrypted such that only the holders of the correct cryptographic keys can access the information in it. Blockchains are thus private.
An emergent property of this structured and shared data is that it eliminates the need for trust brokers between parties who require access to data. Even if not all data in a blockchain can be accessed due to privacy constraints, each stakeholder can prove with mathematical certainty that they are in possession of an exact and unmodified copy of the historical data stream. Everyone has equal information, and well-constructed blockchains ensure that all stakeholders can see all the data required to audit the transactions on the chain. The decentralized and immutable nature of blockchain implementations combined with this transparency means that they convey trust.

Additional rules, often referred to as smart contracts, can be built into these decentralized, immutable, private, and trusted ledgers to regulate how the data can be used. Smart contracts are not a core feature of every blockchain, but are often central to their use in the complex world of healthcare. These contracts benefit from the properties of the blockchain: once set, a smart contract built into a blockchain is immutable and can be trusted to operate the same way, using trusted information shared equally between all parties, indefinitely. Kristin Lauter, Principal Research Manager at Microsoft, has said "you can propose any crazy encryption you want and say it's secure. Why should anyone believe you?" (Molteni, 2017). Blockchain technology answers: bitcoin, a high-value implementation of blockchain, has been open for years to hackers with a lot to gain but remains secure. This cannot be construed as a guarantee of future performance, but it does provide some measure of confidence.

It seems important to add, given the frenzy in the press regarding blockchain technologies (Panetta, 2017), that blockchains are tools with useful properties that may be applicable in many areas, but cannot by themselves solve the panoply of issues endemic to our institutions. Even with perfect technology, the information being put onto a blockchain can still contain faults, and any rules for accessing and adding new information to blockchains must first be created and agreed to by the holders of the consensus. The benefits of applying blockchain technology can be fully realised only after investment in careful technical and administrative planning that includes all stakeholders.

\section{Examples of Blockchain Technology Applications to Healthcare}

In general, blockchain technology is best suited to projects where: 


\section{An Introduction to Blockchain Technology in the Healthcare Sector}

\section{Mark A. Engelhardt}

\section{There multiple stakeholders contributing.}

2. More trust is required between parties than currently exists.

3. There an intermediary that could be removed or omitted to increase trust or efficiency.

4. There is a need for reliable tracking of activity.

5. There is a need for data to be reliable over time.

An examination of some real applications may give a better understanding of how blockchain technology works in healthcare, what it offers, and the current state of the industry. The following specific examples have been chosen to clarify concepts, and do not indicate the importance of one approach versus another. A full exploration of blockchain technology companies throughout the health sector is beyond the scope of this review, but an attempt has been made to identify a collection of international and noteworthy examples.

\section{Busting prescription drug fraud}

Prescription drug fraud is a well-defined challenge to which blockchain technology can be applied. In one example, the blockchain company Nuco attempts to address three common exploits employed to execute prescription fraud: modifying numbers to change the prescription itself, duplication of prescriptions (e.g., photocopying), and so-called "doctor shopping" whereby fraudsters visit many doctors to collect as many original prescriptions as possible (Kesem Frank, personal communication, August 24, 2017). To address these problems, experts have called for monitoring programs to be installed that improve access and response time, scan prescription data to flag suspicious purchasing patterns, and can alert physicians and pharmacists (McDonald \& Carlson, 2013). Nuco identifies the problem as an "open-ended loop", meaning that there is incomplete feedback between the prescription writers (physicians) and the prescription fillers (pharmacists). This fragmented communication is the kind of problem blockchain can solve (Figure 2).

Nuco's blockchain-based solution to the prescription fraud problem works as follows: when a prescription is produced by a doctor, a machine-readable code is attached that serves as a unique identifier. This unique identifier is then associated with a block of information including the name of the drug, the quantity, the anonymized identity of the patient, and a timestamp.
(A)

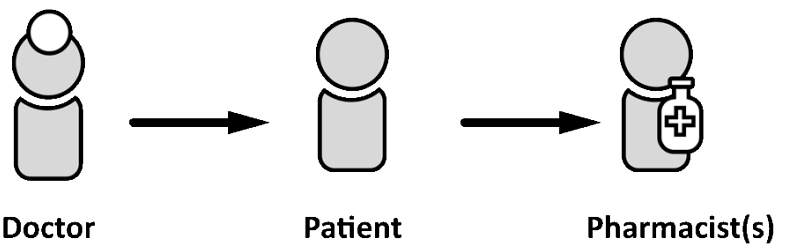

(B)
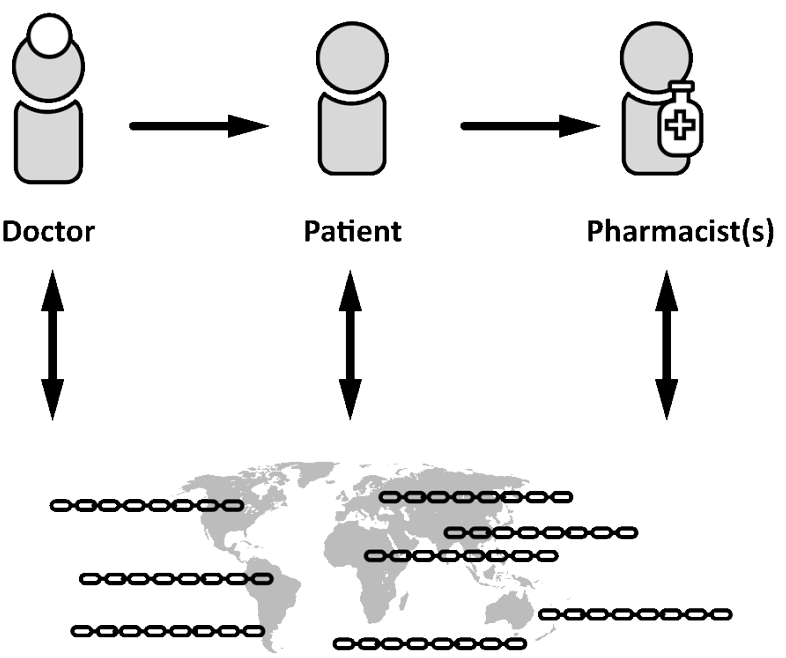

Figure 2. (A) An example of an open-ended loop, where a patient is given a prescription by a doctor, who then delivers it to one (or more) pharmacist(s). A pharmacist has no knowledge of whether the prescription is original, accurate, or previously filled. (B) To close the loop, transactions are stored on blockchains. Each stakeholder can access and add to the blockchains as appropriate. For example, a doctor can add record the original prescription and a pharmacist can check that the prescription is unaltered; a pharmacist can record actions on a prescription, and the doctor or another pharmacist can check its status.

When the prescription is filled by a pharmacist, the symbol is scanned, the attempt to fulfill the prescription is recorded on and compared against the blockchain, and the pharmacist is quickly informed whether the prescription is eligible to be filled and given information to verify its accuracy.

Copies of the blockchain, or distributed ledger, are held by multiple stakeholders in a decentralized network. These stakeholders might include pharmacy chains, insurance providers, auditors, or hospitals, each of whom has a vested interest in solving prescription drug fraud and is large enough to dedicate resources to the com- 


\title{
An Introduction to Blockchain Technology in the Healthcare Sector
}

\author{
Mark A. Engelhardt
}

puting infrastructure required. Due to the encryption of the blockchain information, privacy is maintained when it is passed between stakeholders, and each of the stakeholders can only access information to which they are specifically entitled through the possession of the correct cryptographic keys. Each of the stakeholders can trust that the information they have is accurate because each has an unbroken chain that is identical to the other chains and that they can audit to ensure its integrity.

This solution illustrates an example of a permissioned blockchain, in which only specified parties can read information and transact. It is one of two common broad implementations of blockchain technology; the other is public chains, of which an example is given below.

The Nuco solution integrates on top of existing patterns of usage and uses existing technologies (e.g., the pharmacist only requires a smartphone or similar device to read the unique identifier), providing interoperability with existing protocols. Interoperability will be an important consideration as new blockchain projects interface with both current and new technology for information storage.

HealthChainRx and Scalamed are also working on blockchain solutions to combat prescription fraud and are close to releasing solutions. Both have expressed a strong desire and emphasis on giving patients control over their data, including the ability to authorize who can use it and how (Dave Evans and Tal Rapke, personal communications, August 2017).

Scalamed plans to adopt a public blockchain rather than a permissioned chain (see Nuco, above), which presents an opportunity to differentiate between these two approaches (Tal Rapke, personal communication, August 27, 2017). In public blockchains, storage and maintenance of the blockchain is not restricted to trusted stakeholders. Instead, anyone who participates is remunerated for handling the encrypted data structure. The blockchain is decentralized across many public nodes that work together to verify and process transactions, resulting in trust that the chain is accurate. They do this without the ability to decrypt private data. The choice of one of these different models, permissioned versus public, is a fundamental decision made early in any blockchain project.

\section{Patient-centred medical records}

If there is a common undercurrent that runs through almost all blockchain technology companies working in the health sector, it is the desire to enable people to exercise more personal control over the data collected about them. Physicians are already inundated with more information than they can deal with, and much, much more is coming. A blockchain solution can lighten this burden on the doctor by creating a higher level of organization, accessibility, and amenability to timesaving digital tools while also further engaging the patient in their own care.

As an initial project, Medicalchain has tackled hospital discharge summaries, which include a summary of treatment and necessary follow-up care. Hospitals have incentive to both ensure these documents are free of liability-creating errors and process them quickly to free up beds for the next patient in the queue. Currently, information is siloed: transferring records over municipal boundaries can require written requests, and problems with duplication of data, fraud, and inaccessible data are rampant (Mo Tayeb, personal communication, $\mathrm{Au}-$ gust 25,2017$)$. Medicalchain has introduced a digitized solution that leads doctors through a structured discharge process that reduces errors and omissions and speeds up review by senior staff. They are currently moving this system to a blockchain, which will enable efficient decentralized sharing of data between stakeholders (e.g., hospitals in different networks and health insurance providers) who will be able to trust that the patient data is private due to encryption and historically accurate due to the immutable nature of the blockchain.

More ambitiously, Medicalchain is currently also developing a permissioned blockchain shared across a network of trusted international healthcare institutions to help patients receive care internationally without complicated collection and transfer of medical records (Mo Tayeb, personal communication, August 25, 2017). Their proposed solution for enabling international blockchains is an opportunity to discuss another important concept: on-chain versus off-chain storage of information. Some jurisdictions do not allow private healthcare data to be stored externally. How then can one construct an international shared data structure? The answer may lie in the same type of cryptographic signature that enables each block of the blockchain to uniquely identify the block that it follows. Similarly, each block can contain cryptographic signatures of remotely stored documents that can be used to prove that a document has not been changed in any way. Data can be kept in each patient's home jurisdiction, and then, when transferred by the patient, proven through signatures recorded and shared through the 


\title{
An Introduction to Blockchain Technology in the Healthcare Sector
}

\author{
Mark A. Engelhardt
}

blockchain to be the complete and accurate record of the patient's medical history. In this scenario, only proof that the document is genuine is stored internationally on the blockchain; the actual documents can sit (in encrypted form) in home jurisdictions until the owner of the data (the patient) decides to share them. Storing cryptographic signatures in this way is known as "off-chain storage" and is a common theme in blockchain technology for the health sector, both to deal with regulatory hurdles and due to the prevalence of large data files such as imaging data, the inclusion and sharing of which on the blockchain precludes a streamlined solution.

Healthcoin, an initiative that first developed a blockchain-based solution for helping people work together to improve diabetes symptoms has since expanded their vision towards building a system to construct a global electronic health record system. They identify a value proposition for patient-centred information control that consists of three principles: 1) give the complete data to the user, 2) allow the user to channel their data to its best use, and 3) allow users to broadcast outcomes with mechanisms in place to certify the broadcasted information (Diego Espinosa, personal communication, August 28, 2017). Healthcoin sees themselves as not being in the healthcare business so much as the data sharing business, with the patient sitting at the control panel.

This is a busy space, and analogous projects to connect patient information between stakeholders are being attempted by numerous other players, including BurstIQ, Factom, GemOS, HealthCombix, MedRec, Patientory, and SimplyVital. Even IBM's Watson is getting into the game (Byers, 2017). Patientory, with a solution that attempts to bridge existing electronic medical record systems in the United States, appears to be the closest to having a real product in the hands of patients (Patientory, 2017).

BurstIQ presents a vision of what can be done once blockchain technology becomes the major medium to store patients' data. They see the future of care at the junction of precision medicine, delivering treatments specific to a particular patient's needs, and machine learning, where artificial intelligence is used to learn from health trends and particular patients' histories (Frank Ricotta, personal communication, August 25, 2017). BurstIQ aims to integrate data streams to gain new insights into individual best health outcomes and help people realize them.
The overt shift to patient responsibility over their own data in these blockchain-based solutions represents a significant change. HealthCombix, in collaboration with PointNurse, is attempting to address this by introducing a nurse-mediated layer to make sure that the data that ends up in the immutable blockchain record is accurate, that it has been transferred correctly to the patient, and that the patient understands how to curate, update, and control access to their records (Cyrus Maaghul, personal communication, August 25 2017.) Another differentiating feature of HealthCombix is their plan to tie their system into a specialized hardware component that can be used to reliably monitor patients and introduce quality records to the blockchain. Bowhead is another initiative interested in using a hardware component to feed trusted information to a blockchain.

Given that these solutions are developed in parallel and in the absence of standards, a new interoperability problem emerges. QBRICS and Nuco (Aion) have initiated projects to develop blockchain-based technologies to translate and consolidate information from multiple sources to reconstruct patient data fragmented across platforms.

\section{Connecting the dental industry}

The dental industry is a highly fragmented market consisting of many independent practitioners. Dentacoin is an initiative that aims to use blockchain technology to connect dentists, patients, and suppliers (manufacturers and laboratories) globally. Phase I of their project was the implementation of a review platform that relies on the immutability and decentralization of blockchains and the transparency and reliability of blockchain-bound smart contracts to create trust in the review process. Desirable actions, such as writing a review, are rewarded by transferring cryptocurrency to the patient, which can then be used to purchase dental services from participating practitioners. Dentists are rewarded for participating through access to market research and cryptocurrency accepted by manufacturers. Dentacoin is banking on the trust and decentralization inherent in blockchains to enable an economy of scale to develop between the participating parties, without requiring additional brokers to manage the interactions between each individual piece of the network. Of note is that this blockchain technology endeavour is dipping its toes into real waters: they already have two proof-ofconcept clinics that accept payments in the Dentacoin currency, and several dozen real practices registered for their review platform (Donika Kraeva, personal commu- 


\title{
An Introduction to Blockchain Technology in the Healthcare Sector
}

\author{
Mark A. Engelhardt
}

nication, August 28, 2017). Future phases of the Dentacoin project plan to use their incentive strategy to encourage patients to educate themselves about dental care, set up insurance contracts between patients and dentists that reward patients who perform a minimum of dental maintenance, and to serve as a patient health record, analogous to the patient records covered in the previous section.

The creators of Dentacoin chose to implement a public blockchain because they felt that a more centralized private blockchain would be less trustworthy due to the more limited number of verifiers ensuring transaction fidelity. As with most initiatives mentioned in this article, they favour the storing of private information off chain (see Medicalchain, above).

\section{Key Additional Areas That May Benefit From Blockchain Technology Integration}

\section{Blockchain technology may revolutionize medical research and individual care}

The storage and sharing of health information presents an enormous challenge, including some important risks to privacy, and fantastic opportunities, including the potential to develop a practical understanding the health of unique individuals instead of generic humanity. Blockchain technology companies are diving into this space and promising a new era of research and discovery propelled by analysis of aggregated longitudinal health information from individuals in the context of that from the population at large, and by a new ability for researchers to access data they need to gain new insights.

As the decreasing cost of whole genome sequencing approaches $\$ 1000$ USD, and still meaningful but somewhat less complete analysis even cheaper, the collection of this data has become increasingly common. As an example of the scale of experiment possible in the past couple of years, one recent study employed whole genome sequences of over a thousand participants (Lippert et al., 2017); in another, two hundred thousand participants contributed genome-wide markers (Lo et al., 2016). The application of this scale of data is potentially revolutionary. The Lo study, for example, found genetic correlations with psychiatric data that may have been impossible to locate with fewer markers. Currently, finding large data sets to better the understanding of interactions between disease and other traits and aspects of human lives is a difficult process filled with many obstacles and much paperwork and bureaucracy. Future understanding of human health may benefit enormously if the data now being accumulated by humans around the world can be made easily accessible to researchers. This must be done while adhering to ethical standards and with maintenance of privacy through effective anonymization and ownership of the data by the individual whom it describes, including the ability to grant and revoke access to it. There is evidence that people want this control, and also that many want their data to be useful: a study of research participants receiving whole genome sequence results expressed a strong desire to receive all results, including the raw data, and to maintain the privacy of the data; also, about a third of them consented to sharing their data (Sanderson et al., 2016). Although there is evidence that some incentives may be required (Pevnick et al., 2016), perhaps with the right communication and protections in place, even more people would be willing to contribute their data to the common good.

As with many aspects of this nascent industry, it will be important to get things right: if privacy and ownership concerns cannot be addressed, the willingness of people to contribute their information may evaporate. Operators in this space are aware of the challenge and they are attempting to grapple with it (Jagadeesh et al., 2017). Encryption and keyed access are a first level of protection, but more work is necessary before solutions are ready to be rolled out widely. It is not a simple problem to store private information into a public space, maintain control of who can access it and how it is applied, and at the same time deal with real-world problems such as key loss and changes in an individual's ability to manage their own data, not to mention navigate the process of carefully defining who should have access to what information and under what circumstances (Tanner, 2013).

Why is blockchain technology an interesting tool for this kind of sharing? In addition to the baseline level of anonymization afforded by the encryption of data (but which non-blockchain solutions could also employ), there are several reasons. The first is the immutability of the data: once stored, data for research can be trusted not to change. Second, storage would be transparent: it would be clear to participants what data was and was not available, and replication of studies to verify results would be more straightforward, and there is good evidence that closer monitoring of studies is warranted (Chan et al., 2004; Dwan et al., 2013). Third, with tested and tried smart contracts in place, owners of the data could have confidence that they control their own 


\title{
An Introduction to Blockchain Technology in the Healthcare Sector
}

\author{
Mark A. Engelhardt
}

data and could both grant and revoke access to it in anonymized form to enable research. The immutability of smart contracts due to their inclusion in the blockchain is no small thing: it provides confidence that once a relationship is established it will not be altered, and, as it continues to work as promised, that any contract is secure versus malfeasance.

The focus here has been on the collection of genetic information from our personal genomes, but this is not the only new stream of information that could contribute vast amounts of data to understanding our individual human health. An increasing body of evidence suggests that our microbiome contains information about our personal health, and sequencing efforts are already collecting mountains of bacteriological data (Lynch \& Pedersen, 2016; Zhernakova et al., 2016). Also, with the advent of the Internet of Things, an explosion of devices is collecting longitudinal data about all aspects of our lives, such as heart rate, step cadence, exercise frequency, vocabulary complexity, diet - almost anything that can be imagined. Clearly, there are privacy issues here that must be considered, but this data, too, could be verified, or at least assigned confidence levels, and used to assess current health and help to inform life decisions for health maintenance and improvement.

\section{Government}

Governments are eager to determine whether the costsaving promises of blockchain technology can be realized, and at the same time to encourage patient empowerment and advance medical research and care. In Canada, a Nuco-Deloitte collaboration has engaged with a publically-funded research institute to provide a solution that enables individuals to participate in genetic research, due to be announced in late 2017 (Kesem Frank, personal communication, August 24, 2017). In the United States, The Illinois Department of Financial and Professional Regulation is partnering with healthcare firm Hashed Health to build solutions that take advantage of blockchain and distributed ledger technologies to improve the efficiency and accuracy of cross-state medical licensure (Hashed Health, 2017). The United Arab Emirates and Estonia have also made investments in storing medical health records using blockchain technology (Anderson, 2016; Hankewitz, 2016). These are just a few examples of recent announcements, and the momentum is growing.

Blockchain technology is a fledgling endeavour and still must be aligned with current policies and procedures, especially in the healthcare industry. Recognizing that working within the strictures of government is a significant hurdle all on its own, the National Research Council Canada's Industrial Research Assistance Program (NRCIRAP) has embarked on an experiment that uses blockchain technology (with its attendant immutability, decentralization and transparency) to organize and disseminate public data about its activities and the companies it serves (National Research Council of Canada, 2017). This is viewed as an achievable program that will demonstrate that a public blockchain can be used to hold government data, with a view to learning about, confronting, and addressing administrative hurdles to the framework, and ultimately lay down a path for more complicated data projects (e.g., health data) in the future (David Lisk, personal communication, August 29, 2017). Projects like this one may help to establish blockchain technology as an effective method to record and share government data and serve as an important building block for more sensitive initiatives in the future.

\section{Auditing}

As Brian Behlendorf, Executive Director of the Hyperledger project, on meeting the sustainable development goals of the World Economic Forum, put it on a recent Hashed Health (2017) podcast:

"Every [goal] involves a metric; every metric, in order to actually know if we are making progress against it or not, needs to come out of an accounting system of some sort, and the best way we know today to build an accounting system that is trustworthy, that is decentralizable ... is with blockchain technology."

Effective and trusted tracking of transactional information at each step of a process in a transparent and immutable way is an over-arching trait of blockchain implementations. Therefore, the idea of auditing intersects much of what has already been discussed. One can imagine many instances where clear auditing of records in healthcare would be advantageous, including such examples as checking medical practitioner credentials, tracking and reconciling errors or ambiguities in patient data, and verifying insurance claims. One example of an initiative that tries to address some of these issues is Pokitdok, which has partnered with Intel to build a blockchain-based solution that provides identity management to validate every partner in a transaction (Miller, 2017). Two examples of what Pokitdok hopes this might enable are near-instant billing and insurance claim resolution, and instant auditing of pharmaceutical supply chains and provenance. iSolve is another company working in this space, and among other projects is working on end-to-end blockchain solutions to track medication distribution. 


\title{
An Introduction to Blockchain Technology in the Healthcare Sector
}

\author{
Mark A. Engelhardt
}

There are situations where the importance of careful tracking becomes painfully clear. Counterfeit and fraudulent medication is a growing problem, especially in parts of the world where regulation and cooperation between governments is lacking (McLaughlin, 2012). Detailed and trustworthy pharmaceutical provenance and chain-of-custody information could be built into a blockchain solution, such that local distributors and consumers could audit their own supply and combat fraudulent practices such as relabelling of expiration dates and counterfeiting (Buckley \& Gostin, 2013; Khan \& Khar, 2015; McLaughlin, 2012; Sprink et al., 2016). Pharmaceuticals are part of a much more general case: everything we consume affects our health, and recently major retailers and food companies have announced a collaboration to identify major areas in the global food supply chain that could benefit from tracking through blockchain technology (Aitken, 2017). It is worth bearing in mind that blockchain is not a magical auditing solution that addresses every challenge. It is a tool that can be used for trusted information storage and sharing, but these initiatives will also require systems to enter accurate and complete information in the first place.

\section{Considerations For Future Blockchain Technology Development}

\section{Standards}

Ultimately, standards will be important to guarantee interoperability between blockchains and to establish rules for the safe storage and transfer of information. Currently, development is dominated by prototypes and initial phases of projects with the primary concerns of functionality and proof of concept. A representative of Dentacoin expressed the general sentiment: "at the moment everyone should focus on the progress of existing solutions as well as new ideas and concepts that might not follow any standardization yet" (personal communication, August 28, 2017). That said, it is important to begin thinking about standards, and a standards group (ISO/TC 307) has been set up for blockchain (ISO, 2016). For those who wish to have a voice in the future of blockchain, this may be an important avenue for contribution.

\section{Intellectual property protection and freedom to operate} must be a key consideration for any blockchain technology initiative

These are early days for the use of blockchain technology in health applications, and exciting new ideas are everywhere. At the same time, fast-moving companies and individuals are taking the opportunity to claim broad swaths of the intellectual property space. A quick patent search reveals that the company EITC Holdings, for example, has 63 granted or pending patents in the United Kingdom with priority dates in early-2016 or later; if EITC has been as aggressive in the United States, they will own a significant portion of claims in the blockchain space. Patent applications do not publish for 18 months after the earliest filing date, so the extent of EITC's filing in the United States will not be known for some time. A report by Reuters suggests that EITC plans to file many more (Wagstaff \& Kaye, 2017). Companies including IBM, Mastercard, Fidelity, and Bank of America have also been very active at claiming intellectual property in this area. The extent to which these early patents will be allowed in patent offices and upheld when challenged has yet to be tested. What is clear is that patents are being awarded in the blockchain sector in many worldwide jurisdictions and that forward-looking companies who wish to protect their intellectual property should develop a plan early on, at least to secure their freedom to operate. The effect that the current apparent centralization of control of intellectual property might have on the industry as a whole is unclear, but should be monitored.

\section{Risks}

Blockchain technology is only as good as its users; if low quality or incorrect information is put onto the chain, then what can be trusted through immutability and decentralization is that low quality and incorrect information will remain on the chain. Blockchain and supporting technologies offer many new opportunities, but care must be taken to evaluate the entire implementation, including what happens to information before and after it is on a blockchain. Interoperability solutions will have to be diligent about information that is stored, and include solutions for resolution of discrepancies and assigning confidence to different kinds of information.

Also, the movement to transfer information and control to the patient is laudable, but must be accompanied by education. As stated by Nicole Tay, a researcher in public health (personal communication), if "the whole point was to empower the patient and address the failures of our current system, which rely exclusively on the patient's trust, [and if we create a new system where patients are empowered to control their data but do not know what to do with it and end up engaging others to manage it for them], are we really moving away from a 'trust-based' management system?" 


\title{
An Introduction to Blockchain Technology in the Healthcare Sector
}

\author{
Mark A. Engelhardt
}

Hopefully, the industry will take advantage of the current hype to establish itself, but not stop asking difficult questions. There is a risk that consumers will be drawn in by the golden promise of longer and happier lives care of big data, which may be difficult to turn down even if there are risks. A poor outcome is expected for the blockchain industry if it moves too quickly in the early days and products are pushed out that are not ready. Although there are aspects of blockchain technology that protect against unauthorized access, a large breach of private data through a technical oversight could result in fear of what should instead have held only promise.

One interesting problem is that the ability to access data in the blockchain is through a "key", which is a unique sequence of characters and digits. If a key is lost, then the data it accesses becomes irretrievable. Losing access to a lifetime of health information through the loss of one of these keys is unacceptable, and solutions will have to be implemented to reconnect users with their data. Current solutions to this introduce back doors to accessing the private data on the blockchain, replacing one problem with another.

Another challenge is that, if the decentralization of a blockchain is broken, for example, if one company acquires access to most servers (more than two-thirds with current enterprise methods), then one agent can become the only agent of consensus and can modify the blockchain, contravening the immutability property. New technology for consensus and government regulation surrounding blockchain monopolisation may be necessary to protect against this eventuality.

Finally, a spectre on the horizon is the emergence of quantum computing and its predicted ability to break current encryption methods (Bernstein et al., 2017). It is not clear exactly when this will occur, but within the next decade seems possible (Kobie, 2016). We will have many problems if quantum computing resistant encryption is not solved by then, but if the entirety of one's health data is sitting in blockchains on publically accessible servers, then the privacy of that information will be at risk.

\section{Conclusions}

The application of blockchain technology to healthcare is in its infancy, and there are important challenges to face and big decisions to make going forward. Our societal concept of privacy has evolved in the face of challenges over the past decade and blockchain technology may continue to push at these boundaries, but also promises to deliver great rewards if embraced. If people are enabled to choose for themselves whether to adopt blockchain-based solutions, many may deem the risks of information loss minimal compared to the promise of an overall gain in privacy and control of one's data (assuming no major data breaches). They may be willing to risk even more for the promise of longer and healthier lives by releasing their own data into massive new collections of anonymized population health data, which could then be processed by artificial intelligence to develop personalized healthcare strategies.

The promise of blockchain technology is to enable the efficient sharing of information with stakeholders while ensuring data integrity and protecting patient privacy. Proponents hope that it will bring power to the people and enable them to make positive decisions that improve their health and that of others around the world. They see a world where data is safer than ever before. Skeptics are concerned about the complications beyond the hype; what is envisioned is a massive disruption of the health sector, and there are many installed and invested parties who will act against that change, not to mention ethical, regulatory, and technical details still to figure out.

If the challenges of interoperability continue to be overcome, dependable privacy established, good anonymization protocols developed, and consensus achieved around the kinds of contracts needed to control information, then a new age of healthcare may be around the corner. These are significant challenges, but as described above, companies have already made significant inroads into addressing them even at this early stage. This century's technology giants have already shown us that they are good at using artificial intelligence to learn from data; the same kind of technology is poised to produce disruptive new insights with the kind of data now being produced around health, with privacy and patient control as an important central tenet. Some see this as an important step towards the "health singularity": a transformative event where individualized healthcare is delivered based on a deep understanding of the personal biology of each individual.

The potential of blockchain technology is currently being explored across many healthcare sector implementations. A close watch on the companies mentioned in this review, many of which expect to make major announcements in coming months, would be a good first step to keep apace of developments. The technology (and its marketing) is booming, and care should be 


\title{
An Introduction to Blockchain Technology in the Healthcare Sector
}

\author{
Mark A. Engelhardt
}

taken to look beyond "white papers" and press announcements. Academic literature seems to be lagging, which leaves sources such as the press and critical discussions in online forums such as Reddit as primary options to seek sober second thought. It is an exciting time, with many new applications and implementations being discovered and developed, and full of much promise.

\section{About the Author}

Mark Engelhardt, holds a PhD from Stanford University in the United States and is a partner at Ovodenovo Intellectual Property Consulting, a fullservice patent agency in Ottawa, Canada, where he combines multidisciplinary experience in biological and computational science with experience in intellectual property to help small- and medium-sized companies succeed and to help investors understand technology. He has a passion for non-profit work, and is currently supporting the Ottawa Youth Orchestra Academy as president of their board of directors. Connect through LinkedIn to chat about blockchain technology, intellectual property, nonprofit work, and the interesting places they might intersect in the future.

\section{References}

Aitken, R. 2017. IBM Forges Blockchain Collaboration With Nestlé \& Walmart In Global Food Safety. Forbes, August 22, 2017. Accessed October 1, 2017:

https://www.forbes.com/sites/rogeraitken/2017/08/22/ibmforges-blockchain-collaboration-with-nestle-walmart-for-globalfood-safety/

Anderson, R. 2016. Dubai to Use Blockchain Technology for All Government Documents by 2020. Gulf Business, October 5, 2016. Accessed October 1, 2017: http://gulfbusiness.com/dubai-use-bitcoin-database-technologygovernment-documents-2020/

Arsenijevic, J., Pavlova, M., Rechel, B., \& Groot, W. 2016. Catastrophic Health Care Expenditure among Older People with Chronic Diseases in 15 European Countries. PLoS ONE, 11(7): e0157765. https://doi.org/10.1371/journal.pone.0157765

Avendano, M., \& Kawachi, I. 2014. Why Do Americans Have Shorter Life Expectancy and Worse Health Than Do People in Other HighIncome Countries? Annual Review of Public Health, 35(1): 307-225. https://doi.org/10.1146/annurev-publhealth-032013-182411
Bernstein, D. J., Heninger, N., Lou, P., \& Valenta, L. 2017. PostQuantum RSA. International Workshop on Post-Quantum Cryptography: 311-329. http://dx.doi.org/10.1007/978-3-319-59879-6_18

Buckley, G. J., \& Gostin, L. O. (Eds.). 2013. Countering the Problem of Falsified and Substandard Drugs. Washington, DC: National Academies Press.

https://www.ncbi.nlm.nih.gov/books/NBK202527/

Byers, J. 2017. IBM Watson, FDA Aim to Tackle, Tame Blockchain for Data Exchange. Healthcare Dive, January 11, 2017. Accessed October 1, 2017:

http://www.healthcaredive.com/news/ibm-watson-fda-aim-totackle-tame-blockchain-for-data-exchange/433833/

Canada: Minister of Justice. 2015. Personal Information Protection and Electronic Documents Act. Ottawa: Minister of Justice (Canada).

http://laws-lois.justice.gc.ca/eng/acts/P-8.6/FullText.html

Chan, A. W., Hróbjartsson, A., Haahr, M. T., Gøtzsche, P. C., \& Altman, D. G. 2004. Empirical Evidence for Selective Reporting of Outcomes in Randomized Trials. JAMA, 291(20): 2457-2465. https://doi.org/10.1001/jama.291.20.2457

Collins, F. S. 2015. Exceptional Opportunities in Medical Science: A View from the National Institutes of Health. JAMA, 313(2): 131-132. https://doi.org/10.1001/jama.2014.16736

Das, R. 2017. Top 5 Reasons Why Every Healthcare Company Should Invest in Blockchain. Forbes, August 8, 2017. Accessed October 1, 2017:

https://www.forbes.com/sites/reenitadas/2017/08/08/top-5reasons-why-every-healthcare-company-should-invest-inblockchain/

de Lusignan, S., Mold, F., Sheikh, A., Majeed, A., Wyatt, J. C., Quinn, T., Cavill, M., Gronlund, T. A., Franco, C., Chauhan, U., Blakey, H., Kataria, N., Barker, F., Ellis, B., Koczan, P., Arvanitis, T. N., McCarthy, M., Jones, S., \& Rafi, I. 2014. Patients' Online Access to Their Electronic Health Records and Linked Online Services: A Systematic Interpretative Review. BMJ Open, 4(9): e006021-e006021.

http://dx.doi.org/10.1136/bmjopen-2014-006021

Dwan, K., Gamble, C., Williamson, P. R., \& Kirkham, J. J. 2013. Systematic Review of the Empirical Evidence of Study Publication Bias and Outcome Reporting Bias - An Updated Review. PLoS ONE, 8(7): e66844. https://doi.org/10.1371/journal.pone.0066844

Hankewitz, S. 2016. Estonia to Protect Patient Records with Blockchain Technology. Estonian World, March 4, 2016. Accessed October 1, 2017.

http://estonianworld.com/technology/estonia-to-protect-patientrecords-with-guardtime-blockchain-technology/

Hashed Health. 2017. Illinois Opens Healthcare Blockchain Development Partnership with Hashed Health. Hashed Health, Press Release, August 8, 2017. Accessed October 1, 2017: https://hashedhealth.com/illinois-opens-healthcare-blockchaindevelopment-partnership-with-hashed-health/

ISO. 2016. ISO/TC 307: Blockchain and Electronic Distributed Ledger Technologies. Geneva: International Organization for Standardization (ISO).

https://www.iso.org/committee/6266604.html 


\section{An Introduction to Blockchain Technology in the Healthcare Sector}

\section{Mark A. Engelhardt}

Spink, J., Moyer, D. C., \& Rip, M. R. 2016. Addressing the Risk of Product Fraud: A Case Study of the Nigerian Combating Counterfeiting and Sub-Standard Medicines Initiatives. Journal of Forensic Science \& Criminology, 4(2): 1-13. https://doi.org/10.15744/2348-9804.4.201

Jagadeesh, K. A., Wu, D. J., Birgmeier, J. A., Boneh, D., \& Bejerano, G. 2017. Deriving Genomic Diagnoses without Revealing Patient Genomes. Science, 357(6352): 692-695. http://dx.doi.org/10.1126/science.aam9710

Kelsey, T., \& Cavendish, W. 2014. Personalised Health and Care 2020: Using Data and Technology to Transform Outcomes for Patients and Citizens: A Framework for Action. London: National Information Board, Department of Health, HM Government. https://www.gov.uk/government/publications/personalisedhealth-and-care-2020

Khan, A. N., \& Khar, R. K. 2015. Current Scenario of Spurious and Substandard Medicines in India: A Systematic Review. Indian Journal of Pharmaceutical Sciences, 77(1): 2-7.

Kitson, A., Marshall, A., Bassett, K., \& Zeitz, K. 2013. What Are the Core Elements of Patient-Centred Care? A Narrative Review and Synthesis of the Literature from Health Policy, Medicine and Nursing. Journal of Advanced Nursing, 69: 4-15. http://doi.org/10.1111/j.1365-2648.2012.06064.x

Kobie, N. 2016. Quantum Computing and Its Threat on Encryption and Our Data. Wired UK, October 4, 2016. October 1, 2017: http://www.wired.co.uk/article/quantum-computers-quantumsecurity-encryption

Lippert, C., Sabatini, R., Maher, M. C., Kang, E. Y., Lee, S., Arikan, O., Harley, A., Bernal, A., Garst, B., Lavrenko, V., Yocum, K., Wong, T., Zhu, M., Yan., W.-Y., Chang, C., Lu, T., Lee, C. W. H., Hicks, B., Ramakrishnan, S., Tang, H., Xie, C., Piper, J., Brewerton, S., Turpaz, Y., Telenti, A., Roby, R. K., Och, F. J., \& Venter, J. C. 2017. Identification of Individuals by Trait Prediction Using WholeGenome Sequencing Data. Proceedings of the National Academy of Sciences, 114(38): 10166-10171. http://doi.org/10.1073/pnas.1711125114

Lo, M.-T., Hinds, D. A., Tung, J. Y., Franz, C., Fan, C.-C., Wang, Y., Smeland, O. B., Schork, A., Holland, D., Kauppi, K., Sanyal, N., Escott-Price, V., Smith, D. J., O'Donovan, M., Stefansson, H., Bjornsdottir, G., Thorgeirsson, T. E., Stefansson, K., McEvoy, L. K., Dale, A. M., Andreassen, O. E., \& Chen, C.-H. 2016. Genome-Wide Analyses for Personality Traits Identify Six Genomic Loci and Show Correlations with Psychiatric Disorders. Nature Genetics, 49(1): 152-156. https://dx.doi.org/10.1038\%2Fng.3736

Lynch, S. V, \& Pedersen, O. 2016. The Human Intestinal Microbiome in Health and Disease. New England Journal of Medicine, 375(24): 2369-2379. http://doi.org/10.1056/NEJMra1600266

Mattei, T. A. 2017. Privacy, Confidentiality, and Security of Health Care Information: Lessons from the Recent WannaCry Cyberattack. World Neurosurgery, 104: 972-974. https://doi.org/10.1016/j.wneu.2017.06.104

McDonald, D. C., \& Carlson, K. E. 2013. Estimating the Prevalence of Opioid Diversion by "Doctor Shoppers" in the United States. PLOS ONE, 8(7): e69241.

https://doi.org/10.1371/journal.pone.0069241
McKinsey \& Company. 2011. Big Data: The Next Frontier for Innovation, Competition, and Productivity. New York: McKinsey Global Institute.

https://www.mckinsey.com/business-functions/digitalmckinsey/our-insights/big-data-the-next-frontier-for-innovation

McLaughlin, E. K. 2012. Counterfeit Medicine from Asia Threatens Lives in Africa. The Guardian, December 23, 2012. Accessed October 1, 2017: https://www.theguardian.com/world/2012/dec/23/africacounterfeit-medicines-trade

Mold, F., De Lusignan, S., Sheikh, A., Majeed, A., Wyatt, J. C., Quinn, T., Cavill M., Franco, C., Chauhan, U., Blakey, H., Kataria, H., Arvanitis, T. N., \& Ellis, B. 2015. Patients' Online Access to their Electronic Health Records and Linked Online Services: A Systematic Review in Primary Care. British Journal of General Practice, 65(632): e141-e151. https://doi.org/10.3399/bjgp15X683941

Mold, F., Ellis, B., De Lusignan, S., Sheikh, A., Wyatt, J. C., Cavill, M., Michalakidis, G., Barker, F., Majeed, A., Quinn, T., Koczan, P., Avanitis, T., Gronlund, T. A., Franco, C., McCarthy, M., Renton, Z., Chauhan, U., Blakey, H., Kataria, N., Jones, S., \& Rafi, I. 2012. The Provision and Impact of Online Patient Access to their Electronic Health Records (EHR) and Transactional Services on the Quality and Safety of Health Care: Systematic Review Protocol. Informatics in Primary Care, 20(4): 271-82.

Molteni, M. 2017. To Protect Genetic Privacy, Encrypt Your DNA. Wired, August 23, 2017. Accessed October 1, 2017: https://www.wired.com/story/to-protect-genetic-privacy-encryptyour-dna/

National Research Council of Canada. 2017. "Workin' on the Chain Gang": Doing Business on the Blockchain. Ottawa: National Research Council Canada.

https://www.nrc-cnrc.gc.ca/eng/stories/2017/blockchain.html

Panetta, K. 2017. Top Trends in the Gartner Hype Cycle for Emerging Technologies, 2017. Gartner, August 15, 2017. Accessed October 1, 2017:

http://www.gartner.com/smarterwithgartner/top-trends-in-thegartner-hype-cycle-for-emerging-technologies-2017/

Patientory. 2017. Patientory to Integrate Dash Payments Using BlockCypher Web Services. Patientory, August 24, 2017. Accessed October 1, 2017:

https://patientory.com/2017/08/24/patientory-integrate-dashpayments-using-blockcypher-web-services/

Pevnick, J. M., Fuller, G., Duncan, R., \& Spiegel, B. M. R. 2016. A LargeScale Initiative Inviting Patients to Share Personal Fitness Tracker Data with their Providers: Initial Results. PLoS ONE, 11(11): e0165908.

http://dx.doi.org/10.1371/journal.pone.0165908

Miller, R. 2017. PokitDok Teams with Intel on Healthcare Blockchain Solution. TechCrunch, May 10, 2017. October, 1, 2017: https://techcrunch.com/2017/05/10/pokitdok-teams-with-intelon-healthcare-blockchain-solution/

Sanderson, S. C., Linderman, M. D., Suckiel, S. A., Diaz, G. A., Zinberg, R. E., Ferryman, K., Wasserstein, M., Kasarskis, A., \& Schadt, E. E. 2016. Motivations, Concerns and Preferences of Personal Genome Sequencing Research Participants: Baseline Findings from the HealthSeq Project. European Journal of Human Genetics, 24(1): 14-20.

http://doi.org/10.1038/ejhg.2015.118 


\section{An Introduction to Blockchain Technology in the Healthcare Sector}

Mark A. Engelhardt

Schumacher, A. 2017. Blockchain \& Healthcare - 2017 Strategy Guide. Munich: Axel Schumacher.

Stewart, M. 2001. Towards a Global Definition of Patient Centred Care: The Patient Should Be the Judge of Patient Centred Care. BMJ, 322(7284): 444-445.

https://doi.org/10.1136/bmj.322.7284.444

Suberg, W. 2017. Alibaba Deploys Blockchain to Secure Health Data in Chinese First. The Cointelegraph, August 18, 2017. Accessed October 1, 2017:

https://cointelegraph.com/news/alibaba-deploys-blockchain-tosecure-health-data-in-chinese-first

Tanner, A. 2013. Harvard Professor Re-Identifies Anonymous Volunteers In DNA Study. Forbes, April 25, 2013. Accessed October 1, 2017:

http://www.forbes.com/sites/adamtanner/2013/04/25/harvardprofessor-re-identifies-anonymous-volunteers-in-dna-study/

The World Bank. 2015. World Development Indicators: Health Expenditure Per Capita (Current US\$). The World Bank. Accessed October 1, 2017:

https://data.worldbank.org/indicator/SH.XPD.PCAP

Tieu, L., Schillinger, D., Sarkar, U., Hoskote, M., Hahn, K. J., Ratanawongsa, N., Ralston, J. D., \& Lyles, C. R. 2016. Online Patient Websites for Electronic Health Record Access among Vulnerable Populations: Portals to Nowhere? Journal of the American Medical Informatics Association, 24(e1): e47-e54. https://doi.org/10.1093/jamia/ocw098

United States: Department of Health and Human Services. 2013. HIPAA Administrative Simplification Regulation Text. Washington, DC: U.S. Department of Health and Human Services. https://www.hhs.gov/sites/default/files/hipaa-simplification201303.pdf

Wagstaff, J., \& Kaye, B. 2017. Exclusive: Company Behind Bitcoin "Creator" Sold to Private Investors. Reuters, April 13, 2017. Accessed October 1, 2017:

http://www.reuters.com/article/us-bitcoin-wright-fundexclusive/exclusive-company-behind-bitcoin-creator-sold-toprivate-investors-idUSKBN17F26V

Woodman, J., Sohal, A. H., Gilbert, R., \& Feder, G. 2015. Online Access to Medical Records: Finding Ways to Minimise Harms. British Journal of General Practice, 65(635): 280-281. https://doi.org/10.3399/bjgp15X685129

Zhernakova, A., Kurilshikov, A., Bonder, M. J., Tigchelaar, E. F., Schirmer, M., Vatanen, T., Mujagic, Z., Vila, A. V., Falony, G., VieiraSilva, S., Wang, J., Imhann, F., Brandsma, E., Jankipersadsing, S. A., Joossens, M., Cenit, M. C., Deelen, P., Swertz, M. A., Weersma, R. K., Feskens, E. J., Netea, M. G., Gevers, D., Jonkers, D., Franke, L., Aulchenko, Y. S., Huttenhower, C., Raes, J., Hofker, M. H., Xavier, R. J., Wijmenga, C., \& Fu, J. 2016. Population-Based Metagenomics Analysis Reveals Markers for Gut Microbiome Composition and Diversity. Science, 352(6285): 565-569.

https://doi.org/10.1126/science.aad3369

Citation: Engelhardt, M. A. 2017. Hitching Healthcare to the Chain: An Introduction to Blockchain Technology in the Healthcare Sector. Technology Innovation

Management Review, 7(10): 22-34.

http://doi.org/10.22215/timreview/1111

Keywords: blockchain, health, distributed ledger,

patient-centred care, trust

timreview.ca 


\section{Academic Affiliations and Funding Acknowledgements}
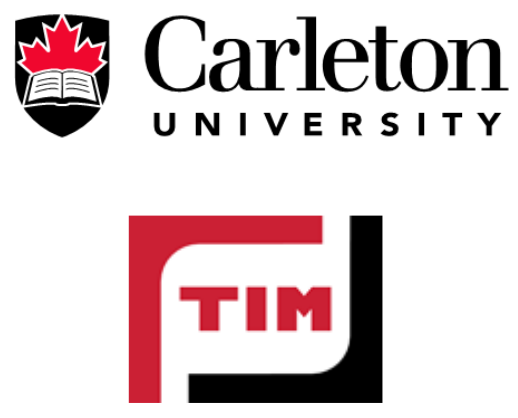

Canadà
Technology Innovation Management (TIM; timprogram.ca) is an international master's level program at Carleton University in Ottawa, Canada. It leads to a Master of Applied Science (M.A.Sc.) degree, a Master of Engineering (M.Eng.) degree, or a Master of Entrepreneurship (M.Ent.) degree. The objective of this program is to train aspiring entrepreneurs on creating wealth at the early stages of company or opportunity lifecycles.

- The TIM Review is published in association with and receives partial funding from the TIM program.

The Federal Economic Development Agency for Southern Ontario (FedDev Ontario; feddevontario.gc.ca) is part of the Innovation, Science and Economic Development portfolio and one of six regional development agencies, each of which helps to address key economic challenges by providing regionallytailored programs, services, knowledge and expertise.

- The TIM Review receives partial funding from FedDev Ontario's Investing in Regional Diversification initiative. 